\title{
Endothelial lipase is upregulated by interleukin-6 partly via the p38 MAPK and p65 NF-кB signaling pathways
}

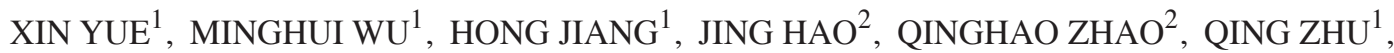 \\ GAOWA SAREN $^{1}$, YUN ZHANG ${ }^{1}$ and XIAOLI ZHANG ${ }^{2}$ \\ ${ }^{1}$ Key Laboratory of Cardiovascular Remodeling and Function Research, \\ Chinese Ministry of Education and Chinese Ministry of Public Health, Department of Cardiology, \\ Qilu Hospital, Shandong University; ${ }^{2}$ Key Laboratory of The Ministry of Education for Experimental Teratology, \\ Department of Histology \& Embryology, Medical College, Shandong University, Jinan, Shandong 250012, P.R. China
}

Received April 21, 2015; Accepted April 15, 2016

DOI: $10.3892 / \mathrm{mmr} .2016 .5457$

\begin{abstract}
To investigate the effects of inflammatory factor interleukin (IL)-6 on the expression of endothelial lipase (EL) and its potential signaling pathways in atherosclerosis, a primary culture of human umbilical vein endothelial cells (HUVECs) was established and treated as follows: i) Control group without any treatment; ii) recombinant human (rh)IL-6 treatment $(10 \mathrm{ng} / \mathrm{ml})$ for $0,4,8,12$ and $24 \mathrm{~h}$; iii) p38 mitogen-activated protein kinases (MAPKs) inhibitor (SB203580, $10 \mu \mathrm{mol} / \mathrm{l})$ pretreatment for $1 \mathrm{~h}$ prior to rhIL-6 $(10 \mathrm{ng} / \mathrm{ml})$ treatment; iv) nuclear factor $(\mathrm{NF})-\kappa \mathrm{B}$ activation inhibitor (pyrrolidine dithiocarbamate, $10 \mathrm{mmol} / \mathrm{l}$ ) pretreatment for $1 \mathrm{~h}$ prior to rhIL-6 $(10 \mathrm{ng} / \mathrm{ml})$ treatment. EL levels were detected by immunocytochemical staining and western blot analysis. Proliferation of HUVECs was detected by immunostaining of proliferating cell nuclear antigen (PCNA) and an MTT assay. p38 MAPK and NF- $\kappa$ B p65 levels were detected by western blotting. The results showed that rhIL- 6 treatment increased EL expression and proliferation of HUVECs. NF- $\kappa \mathrm{B}$ p 65 and MAPK p38 protein levels also increased in a time-dependent manner in HUVECs after rhIL-6 treatment. NF- $\kappa \mathrm{B}$ inhibitor and MAPK p38 inhibitor prevented the effects of rhIL-6 on
\end{abstract}

Correspondence to: Dr Xiaoli Zhang, Key Laboratory of The Ministry of Education for Experimental Teratology, Department of Histology \& Embryology, Medical College, Shandong University, 44 Wenhuaxi Road, Jinan, Shandong 250012, P.R. China

E-mail: zhangxiaoli@sdu.edu.cn

Abbreviations: IL-6, interleukin-6; rhIL-6, recombinant human interleukin-6; NF- $\kappa \mathrm{B}$, nuclear factor- $\mathrm{B}$; PDTC, pyrrolidine dithiocarbamate; MAPKs, mitogen-activated protein kinases; EL, endothelial lipase; HDL, high density lipoprotein; HUVECs, human umbilical vein endothelial cells; EGF, epithelial growth factor; EDTA, ethylenediaminetetraacetic acid; FBS, fetal bovine serum; PBS, phosphate-buffered saline; DAB, diaminobenzidine

Key words: EL, IL-6, p38 MAPK, p65 NF-кB
EL expression. In conclusion, inflammatory factor IL-6 may participate in the pathogenesis of atherosclerosis by increasing EL expression and the proliferation of endothelial cells via the p38 MAPK and NF- $\kappa$ B signaling pathways.

\section{Introduction}

Interleukin (IL)-6 is involved in a number of diseases, such as atherosclerosis $(1,2)$ and rheumatoid arthritis (3). Thus, there are potential clinical benefits to the development of anti-IL-6 agents as therapies for these diseases $(4,5)$. The first example is tocilizumab, which has been approved for the treatment of rheumatoid arthritis (6) and others are currently in clinical trials (7).

Endothelial lipase (EL), a member of the lipase family, is a key enzyme with phospholipase activity that promotes the metabolism and clearance of high-density lipoprotein (HDL) $(8,9)$. HDL cholesterol levels are inversely associated with the risk of atherosclerotic cardiovascular disease (10). Alternative functions of EL include increasing the uptake of apolipoprotein B by endothelial cells and the adhesion of monocytes and macrophages to endothelial cells (11). An animal study demonstrated that the atherosclerotic plaque area in apoE knockout mice that overexpress EL is $<70 \%$ the area of control mice (8). Consequently, EL expression is closely associated with the pathogenesis of atherosclerosis. EL expression is affected by a number factors, such as inflammation, blood pressure and an increased rate of blood flow (12-14). However, the mechanisms that underlie the regulation of EL expression have not been fully elucidated.

The p38 mitogen-activated protein kinase (MAPK) is a member of the MAPK family and is involved in multiple intracellular signal transduction (15). p65 nuclear factor (NF) $\kappa$ B is a member of the NF- $\mathrm{B}$ transcription factor family and is crucial in inflammatory diseases, such as atherosclerosis (16).

The aim of the present study was to stimulate human umbilical vein endothelial cell (HUVEC) culture in vitro with recombinant human interleukin-6 (rhIL-6), to examine its effect on EL expression and its effects on the proliferation of HUVECs. To investigate the regulatory role of p38 MAPK and p65 NF- $\kappa \mathrm{B}$ in this process, HUVECs were pretreated 
with a p38 MAPK inhibitor (SB203580) or an NF-kB inhibitor (pyrrolidine dithiocarbamate, PDTC) prior to rhIL-6 treatment.

\section{Materials and methods}

Reagents. SB203580, protease inhibitor cocktail and epithelial cell growth factor (EGF) were purchased from Sigma-Aldrich (St. Louis, MO, USA); rhIL-6 was obtained from Peprotech (Rocky Hill, NJ, USA). Trypsin and M199 medium were purchased from Hyclone (Logan, UT, USA). Rabbit polyclonal anti-EL primary antibody was purchased from Cayman Chemicals (Ann Arbor, MI, USA; cat. no. 100030). Mouse anti-Von Willebrand Factor (vWF, also known as Factor VIII related antigen; cat. no. ab194405) was purchased from Abcam (Cambridge, MA, USA). The mouse and rabbit IgG-immunohistochemical SABC kit, DAB (diaminobenzidine) kit and the MTT assay kit were purchased from Beyotime Institute of Biotechnology (Beijing, China). Fluorescein isothiocyanate (FITC)- and tetramethylrhodamine (TRITC)-conjugated anti-mouse IgG (cat. nos. ZF-0312 and ZF-0313, respectively) were obtained from Zhongshanjinqiao Biotechnology (Beijing, China); mouse anti-proliferating cell nuclear antigen (PCNA) antibody (cat. no. BM0104) was purchased from Boster Biological Technology, Ltd. (Wuhan, China), and monoclonal anti- $\beta$-actin mouse primary antibody (cat. no. AA128-1) was from Beyotime Institute of Biotechnology. Radioimmunoprecipitation assay (RIPA) lysis buffer and the Bicinchoninic acid (BCA) Protein Assay kit were purchased from Beyotime Institute of Biotechnology. Fetal bovine serum (FBS) was obtained from Yuanpeng Biotech Co. (Jinan, China). Penicillin was from North China Pharmaceutical Co. (Shijiazhuang, China). Streptomycin was obtained from Merro Pharmaceutical Co. (Dalian, China). Polyvinylidene fluoride (PVDF) membranes were from Millipore (Millipore Corporation, Bedford, MA, USA). An electrochemiluminescence kit was purchased from Amersham (GE Healthcare, Barrington, IL, USA).

Ethical approval. This study was approved by the Ethical Committee at the School of Medicine, Shandong University (Jinan, China; permit number: 200800243). Written informed consent for the donation of the umbilical cords used in this study was obtained from the parents of the newborn.

Cell culture. Human umbilical vein endothelial cells were obtained from one fresh umbilical cord $\sim 15-\mathrm{cm}$ long from a newborn infant after maternal cesarean section in the Department of Obstetrics and Gynecology of Qilu Hospital (Jinan, China). Briefly, the umbilical vein was washed with sterile phosphate-buffered saline (PBS) for $5 \mathrm{~min}$. Trypsin-ethylenediaminetetraacetic acid $(0.25 \%$; Gibco; Thermo Fisher Scientific, Inc., Waltham, MA, USA) was injected through the umbilical vein and digested for 10-15 min at room temperature with gentle shaking so that the enzyme solution was in full contact with the vascular wall. The solution was then collected in a 50-ml sterile tube and centrifuged at $100 \mathrm{x} \mathrm{g}$ for $10 \mathrm{~min}$. The supernatant was removed and $20 \%$ FBS cell culture media were added (Hyclone, 20\% FBS, $100 \mathrm{U} / \mathrm{ml}$ penicillin, $100 \mathrm{U} / \mathrm{ml}$ streptomycin and $3 \mu \mathrm{l} / \mathrm{ml} \mathrm{EGF}$ ) to obtain the cell suspension. The cell suspension was transferred to a 6-well culture plate and incubated in an incubator with saturated humidity and $5 \% \mathrm{CO}_{2}$ at $37^{\circ} \mathrm{C}$. The media were changed every 2-3 days. Cells were passaged after they reached $80-90 \%$ confluency. The passaged cells were continuously incubated in 10\% FBS culture media. The purity of HUVECs was determined by immunofluorescence staining of vWF when cells had reached $>90 \%$ confluence.

Cell treatments. Cells were treated after the third passage and divided into 4 groups treated as follows: i) Control group without any treatment; ii) rhIL-6 (10 ng/ml); iii) SB203580 (10 $\mu \mathrm{mol} / \mathrm{l})$ pretreatment for $1 \mathrm{~h}+\mathrm{rhIL}-6$ (10 ng/ml); and iv) PDTC $(10 \mathrm{mmol} / \mathrm{l})$ pretreatment for $1 \mathrm{~h}+\mathrm{rhIL}-6(10 \mathrm{ng} / \mathrm{ml})$ purchased from Beyotime Institute of Biotechnology. Cells from each group $\left(\sim 2 \times 10^{7}\right)$ were collected at $0,4,8,12$ and $24 \mathrm{~h}$ respectively. Before being passaged and treated, coverslips were placed in the culture plates for later use.

Immunocytochemical staining. The coverslips coated with cells were removed and washed in sterile PBS. The cells were fixed in methanol: acetic acid (3:1) for $10 \mathrm{~min}$ and washed in PBS. Then, the cells were immunoblocked with $10 \%$ normal goat serum in $0.01 \mathrm{M}$ PBS containing $0.3 \%$ Triton $\mathrm{X}-100$ at room temperature for $1 \mathrm{~h}$. Primary antibodies against EL (1:100), vWF (1:100) and PCNA (1:100) were then added and incubated in a humidified box at $4^{\circ} \mathrm{C}$ overnight. Biotin-conjugated anti-rabbit IgG (for EL; cat. no. A0277; Beyotime Institute of Biotechnology) or FITC- (for vWF cat. no. ZF-0312) and TRITC- (for PCNA; cat. no. ZF-0313) conjugated anti-mouse IgG were added (for PCNA and vWF) and incubated at $37^{\circ} \mathrm{C}$ for $1 \mathrm{~h}$. Then SABC complex was added and incubated at $37^{\circ} \mathrm{C}$ for $1 \mathrm{~h}$ according to the manufacturer's instructions of the SABC kit and DAB color was developed. Between each step, cells were washed twice in PBS. Negative control staining was performed with nonspecific IgG instead of the primary antibody. Cells were counterstained with hematoxylin and finally sealed with Neutral balsam. For vWF and PCNA, after IgG incubation, they were counterstained with DAPI and sealed with anti-fade (Beyotime Institute of Biotechnology). All coverslips were examined under an Olympus U-LH100HG microscope (Olympus Corporation, Tokyo, Japan).

Western blotting. EL protein levels in HUVECs were detected by western blotting. HUVECs in each group were harvested separately, washed in cold PBS, and homogenized at $4^{\circ} \mathrm{C}$ in lysis buffer containing $10 \mathrm{mM}$ HEPES, $\mathrm{pH} 7.9 ; 10 \mathrm{mM}$ $\mathrm{KCl}, 1.5 \mathrm{mM} \mathrm{MgCl}{ }_{2}, 0.1 \mathrm{mM}$ EGTA, $0.5 \mathrm{mM}$ DTT, $10 \mathrm{mM}$ $\alpha$-glycerophosphate, $0.1 \mathrm{mM}$ sodium vanadate and a protease inhibitor cocktail. After $15 \mathrm{~min}$ of incubation on ice, cell debris was removed by centrifugation at $15,000 \mathrm{x}$ g for $20 \mathrm{~min}$ at $4^{\circ} \mathrm{C}$. Protein concentration was determined by the $\mathrm{BCA}$ assay with bovine serum albumin (Boster Biological Technology, Ltd.) as a standard. Proteins $(40 \mu \mathrm{g})$ were separated on a $10 \%$ sodium dodecyl sulfate-polyacrylamide gel, and then transferred to a polyvinylidene difluoride membrane. After blocking with 5\% (w/v) fat-free milk for $1 \mathrm{~h}$ at room temperature, the membranes were probed with anti-EL $(1: 1,500)$ overnight at $4^{\circ} \mathrm{C}$, followed 
A

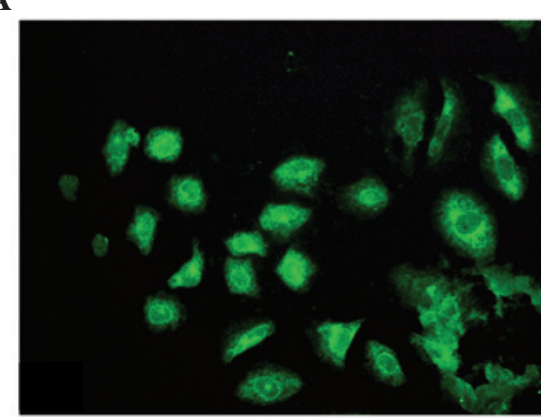

B

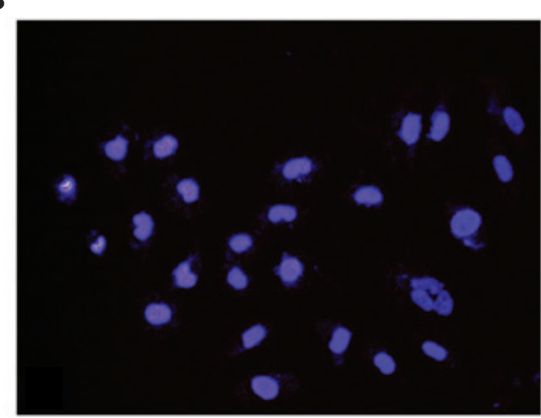

C

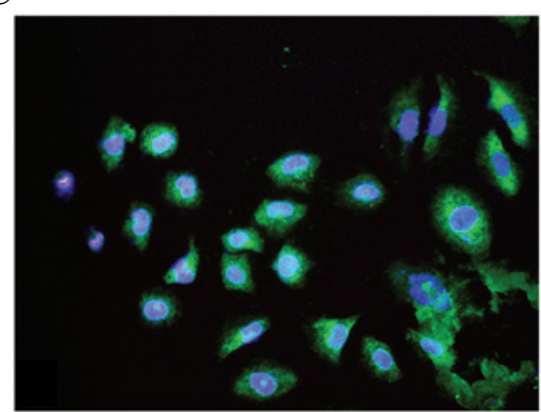

Figure 1. Immunofluorescence staining of vWF was performed to identify human umbilical vein endothelial cells cultured in vitro. The panels show (A) immunostaining of vWF (green), (B) staining for nuclei with DAPI (blue) and (C) a merged image. Over 95\% of the cells were vWF positive. Magnification, $\mathrm{x} 400$. vWF, Von Willebrand factor.

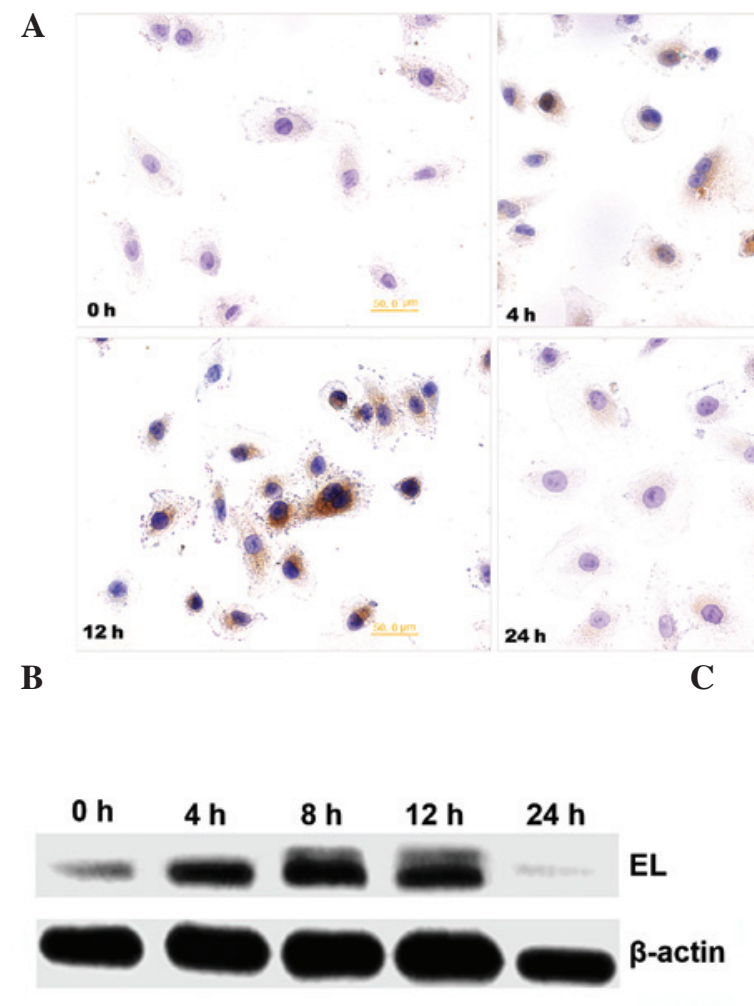

A
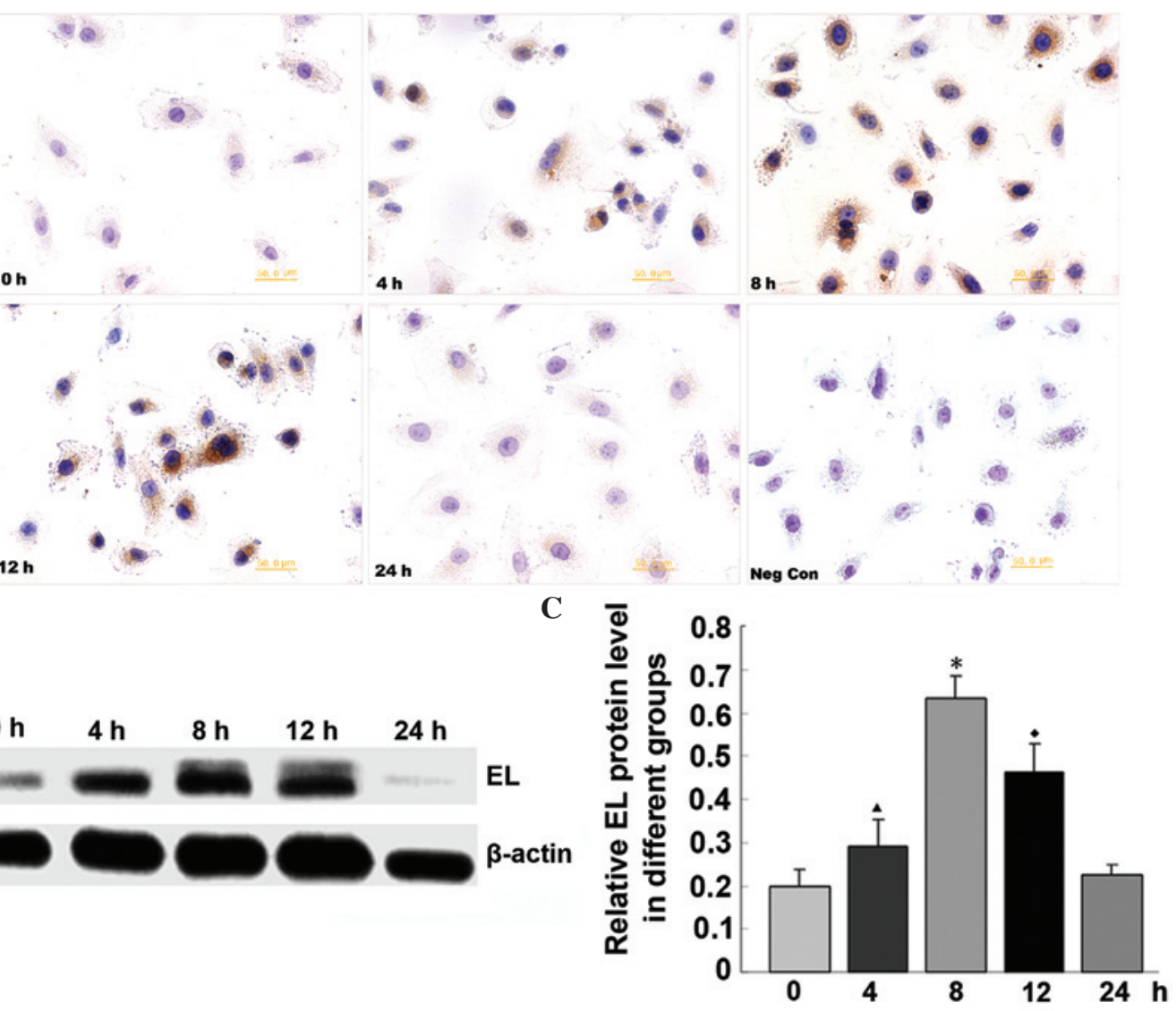

Figure 2. rhIL-6 treatment increased EL expression in HUVECs by immunocytochemical staining and western blotting. (A) Immunocytochemical staining of EL at 0, 4, 8, 12 and $24 \mathrm{~h}$ after rhIL- 6 treatment and the negative control. Magnification, $\mathrm{x} 400$. (B) EL expression by western blotting at $0,4,8,12$ and $24 \mathrm{~h}$ after rhIL-6 treatment. (C) Semi-quantitative analysis of the EL level of the western blotting results. ${ }^{\wedge} \mathrm{P}<0.05$ compared with the $0,8,12$ and $24 \mathrm{~h}$ groups; ${ }^{*} \mathrm{P}<0.05$ compared with $0,4,12$ and 24 h groups; ${ }^{\bullet} \mathrm{P}<0.05$ compared with $0,4,8$ and 24 h group. rhIL-6, recombinant human interleukin-6; EL, endothelial lipase; HUVECs, human umbilical vein endothelial cells.

by incubation with peroxidase-conjugated anti-rabbit IgG (1:500) for $1 \mathrm{~h}$ at room temperature. The interaction was monitored with an electrochemiluminescence kit. $\beta$-Actin was used to monitor the loading amount.

Statistical analysis. Densitometric evaluation of western blotting results were conducted using the Quantity One software, version 4.62 (Bio-Rad Laboratories, Inc., Hercules, CA, USA) with $\beta$-actin as an internal control. Immunocytochemical staining results were analyzed with Image-Pro Plus 5.0 (Media Cybernetics, Inc., Rockville, MD, USA). Data are presented as the mean \pm standard deviation of three separate experiments. Comparisons among groups were conducted using one-way analysis of variance (ANOVA). If the result of ANOVA was statistically significant, then multiple comparison tests between groups were performed using the Student-Newman-Keuls method. $\mathrm{P}<0.05$ was considered to indicate a statistically significant difference. 


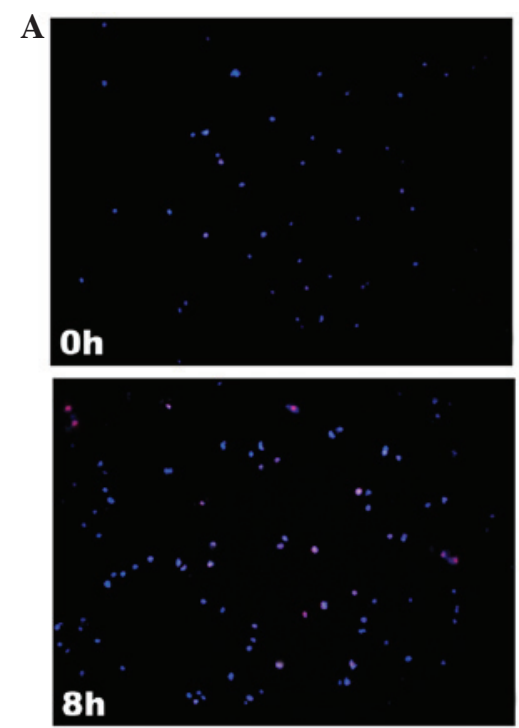

B

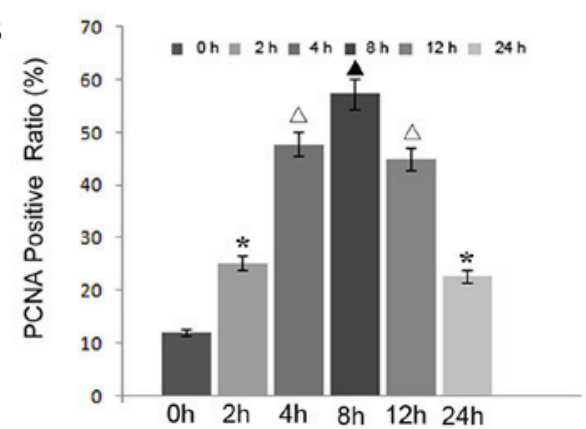

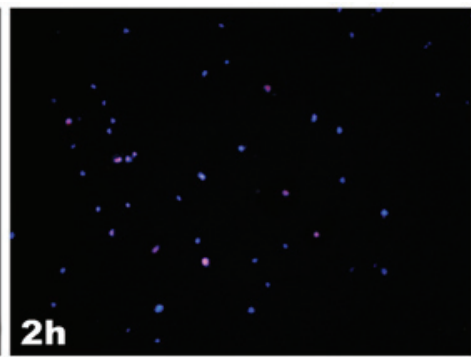
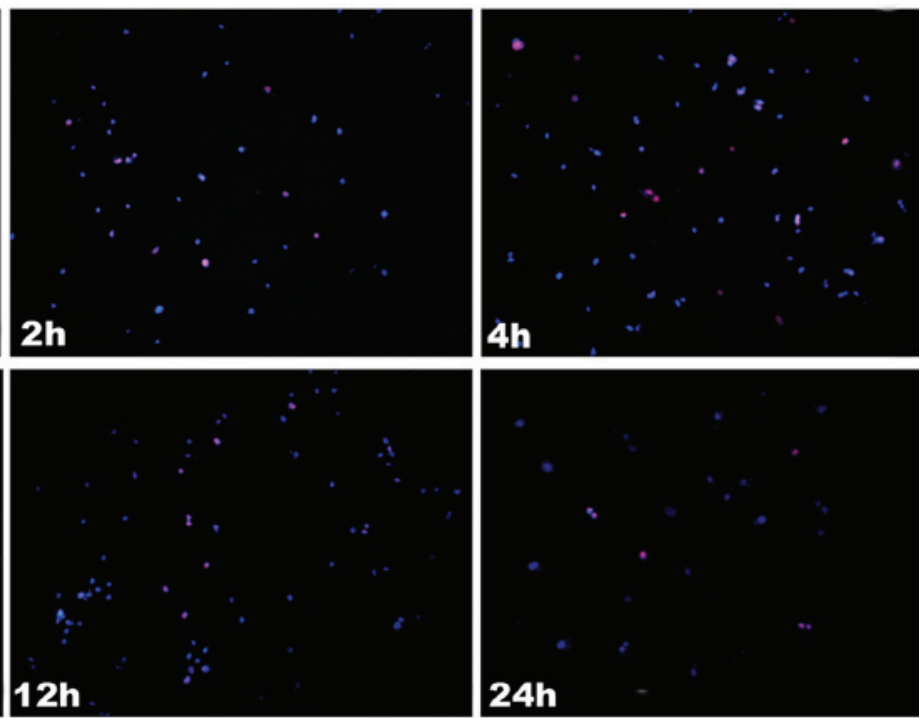

C

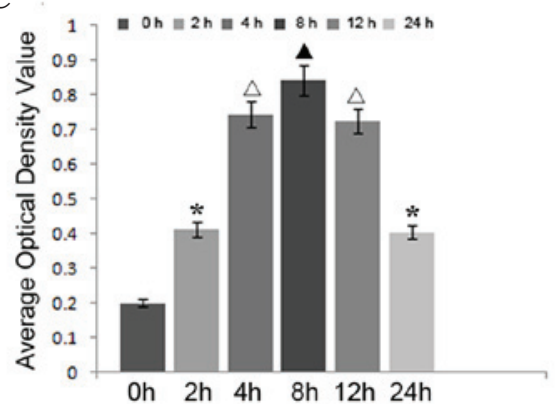

Figure 3. rhIL-6 treatment promoted the proliferation of HUVECs. rhIL-6 treatment was shown to promote HUVEC proliferation by immunofluorescence staining of PCNA and MTT assays. (A) Immunofluorescence staining of PCNA in HUVECs in each group. magnification, x100. (B) Semi-quantitative analysis of the immunofluorescence staining of PCNA. (C) Statistical analysis of the MTT assays for each group. Different symbols indicate statistical significance $(\mathrm{P}<0.05)$. ${ }^{\wedge} \mathrm{P}<0.05$ compared with the $0,2,4,12$ and $24 \mathrm{~h}$ groups; ${ }^{*} \mathrm{P}<0.05$ compared with the $0,4,8$ and $12 \mathrm{~h}$ groups; ${ }^{\wedge} \mathrm{P}<0.05$ compared with the $0,2,8$ and $24 \mathrm{~h}$ groups. rhIL-6, recombinant human interleukin-6; EL, endothelial lipase; HUVECs, human umbilical vein endothelial cells; PCNA, proliferating cell nuclear antigen.

\section{Results}

Isolation and identification of HUVECs. vWF is expressed in HUVECs, this the immunofluorescence staining of vWF was performed to identify HUVECs. The results showed that almost all cells were vWF positive (Fig. 1).

rhIL-6 treatment increases the EL expression in HUVECs. After HUVECs were treated with rhIL-6, EL expression levels increased significantly from $4 \mathrm{~h}$. The effect persisted at significant levels until $12 \mathrm{~h}$ after treatment, and EL expression decreased to basal levels by $24 \mathrm{~h}$. The effect was most significant at $8 \mathrm{~h}$ after rhIL- 6 treatment. After that time, the EL expression level began to decrease up to $24 \mathrm{~h}$ after treatment, when the EL expression level returned to the basal level. The immunocytochemical staining results are consistent with that of western blotting (Fig. 2).

rhIL-6 treatment promotes proliferation of HUVECs. In order to detect the effects of increased EL on HUVECs, immunocytostaining of PCNA and an MTT assay were performed to detect the proliferative activity of HUVECs at 0, 4, 8, 12 and $24 \mathrm{~h}$. The results showed that increased EL promoted HUVEC proliferation (Fig. 3). Similar to the change in the expression levels of EL, the ratio of PCNA-positive cells was highest at $8 \mathrm{~h}$ after rhIL- 6 treatment, after which the ratio reduced. At $24 \mathrm{~h}$ after rhIL-6 treatment, the ratio of PCNA-positive cells ratio remained higher than the original level.

rhIL-6 treatment increases $p 38 M A P K$ and $p 65 N F-\kappa B$ expression in HUVECs. To determine whether IL-6 regulates EL expression through MAPK or NF- $\mathrm{KB}$ signaling pathway, p38 MAPK and p65 NF- $\kappa$ B protein levels at 4, 8 and $12 \mathrm{~h}$ were detected by western blotting. The results showed that $\mathrm{p} 38$ MAPK or $\mathrm{p} 65$ expression changes were consistent with that of EL. That is, after HUVECs were incubated with rhIL-6, p38 MAPK and $\mathrm{p} 65$ NF- $\mathrm{kB}$ expression levels increased significantly at 4,8 and $12 \mathrm{~h}$ (Fig. 4).

p38 MAPK inhibitor SB203580 or p65 NF- $\kappa$ B inhibitor PDTC pretreatment decreases EL expression in HUVECs. To confirm that IL-6 regulates the EL level via the MAPK p38 and P65 NF-kB signaling pathway, HUVECs were pretreated with the p38 MAPK inhibitor SB203580 or P65 NF-kB inhibitor PDTC for $1 \mathrm{~h}$ prior to rhIL-6 treatment. The results showed that EL expression in the rhIL-6+SB203580 and rhIL-6+PDTC groups was significantly decreased at each time point between 4 and $12 \mathrm{~h}$ compared with the IL-6 group ( $\mathrm{P}<0.05$; Fig. 5). 
A

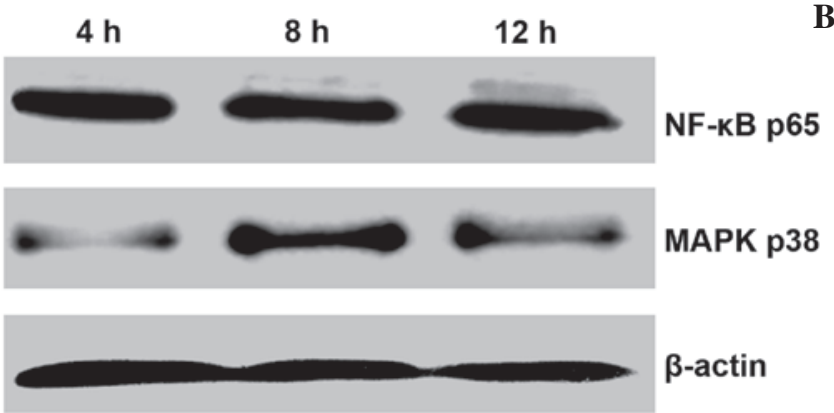

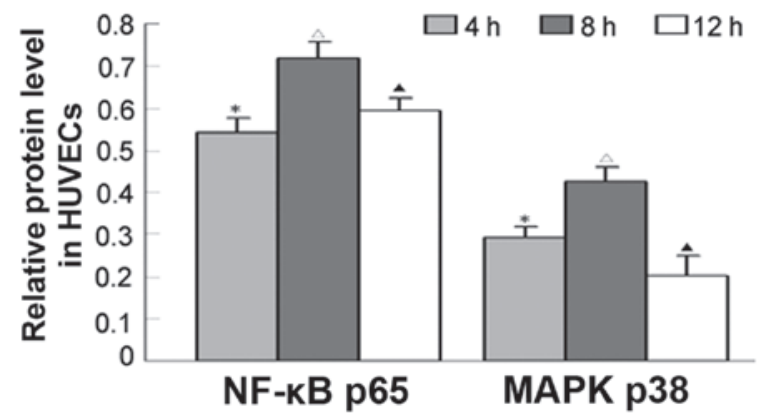

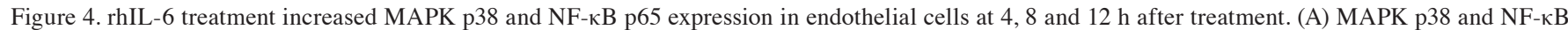
p65 western blotting results at different time points. (B) Semi-quantitative analysis of MAPK p38 and NF-kB p65 levels. ${ }^{\triangle} \mathrm{P}<0.05 \mathrm{compared}$ with the 4 and $12 \mathrm{~h}$ group; ${ }^{*} \mathrm{P}<0.05$ compared with the 8 and $12 \mathrm{~h}$ groups; ${ }^{\wedge} \mathrm{P}<0.05$ compared with the 4 and 8 h groups. rhIL-6, recombinant human interleukin-6; MAPK p38, p38 mitogen-activated protein kinases; $\mathrm{NF}-\kappa \mathrm{B}$, nuclear factor- $\kappa \mathrm{B}$.

A

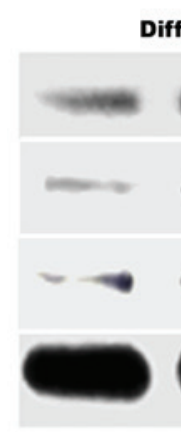

Oh
EL Expression in HUVECs in

Different Groups at Different Time Point

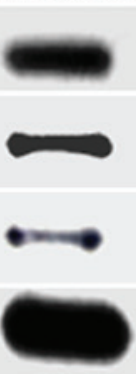

4h

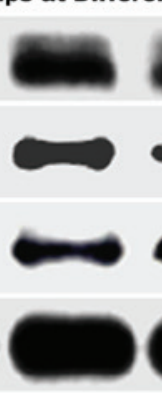

8h

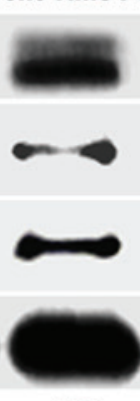

12h
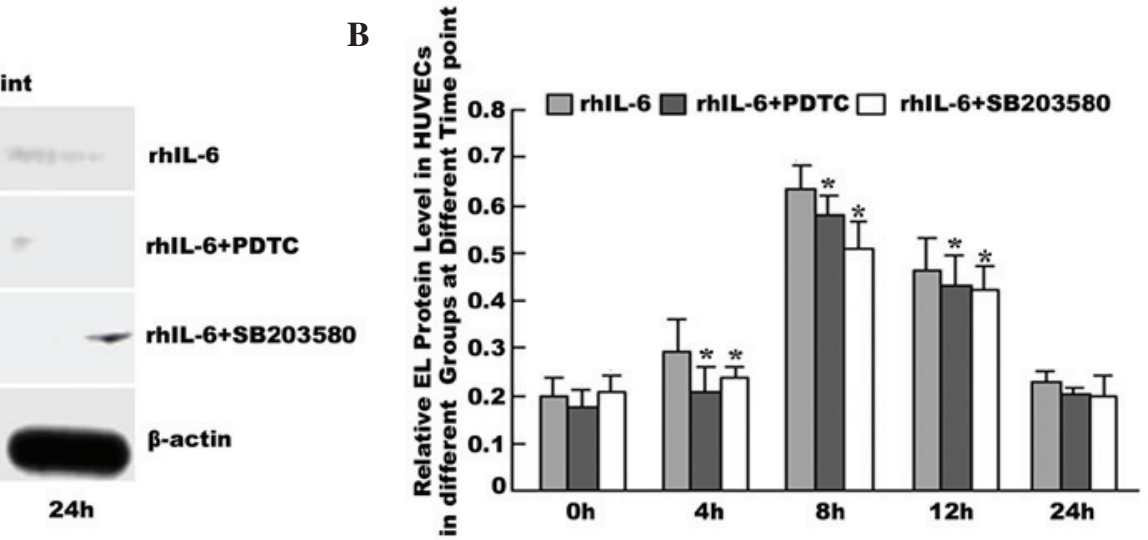

Figure 5. EL expression of HUVECs decreased at 0, 4, 8, 12 and $24 \mathrm{~h}$ in the rhIL-6+SB203580 and rhIL-6+PDTC group compared with the rhIL-6 group. (A) EL expression as determined by western blotting at different time points. (B) Semi-quantitative analysis of the EL level of western blotting results at different time points. "P<0.05 compared with the rhIL-6 group. EL, endothelial lipase; HUVECs, human umbilical vein endothelial cells; PDTC, pyrrolidine dithiocarbamate.

\section{Discussion}

EL was identified in 1999 as part of the triglyceride lipase family of genes (8). Structurally, EL has 45, 40 and $27 \%$ homology with lipoprotein lipase, hepatic lipase and pancreatic lipase, respectively. EL is a critical enzyme in high density lipoprotein (HDL) metabolism by hydrolyzing the phospholipid component (9). The plasma HDL concentration rises significantly in EL gene knockout mice and the overexpression of EL reduces the HDL level markedly while HDL is an independent risk factor for atherosclerosis (8).

EL also has non-enzymatic activities, including mediating the uptake of apolipoprotein B and the adhesion of monocytes or macrophages to the endothelia, which indicates that EL participates in the pathogenesis of atherosclerosis $(11,16,17)$. Recently, accumulating clinical evidence demonstrated that EL is closely associated with coronary atherosclerosis. Badellino et al (18) demonstrated that serum EL concentration in patients with metabolic syndrome was significantly higher than that in the normal population, and this was positively correlated with the development of coronary atherosclerosis. Furthermore, polymorphisms of the gene encoding EL may be associated with the progression of acute coronary syndrome (18-20). Fang et al (21) found that EL expression increases in the endothelial cells of the vascular system in patients with coronary heart disease and this increase is correlated with the severity of the clinical syndrome and also increases the coronary risk scores.

Atherosclerosis is a chronic inflammatory disease, characterized by the interaction of inflammatory mediators and cytokines in vascular endothelia. In 2000, it was demonstrated that IL-1 $\beta$ and tumor necrosis factor- $\alpha$ (TNF- $\alpha$ ) upregulates the mRNA expression of EL in HUVECs in vitro (22). Jin et al (23) confirmed that this was partially mediated through the NF- $\kappa \mathrm{B}$ pathway. Badellino et al (24) and Paradis et al (25) found that IL-6, soluble tumor necrosis factor-R, soluble intracellular adhesion molecule and other cytokines are positively correlated with EL levels (24). These data indicated that inflammation has a significant effect on the expression of EL. The present results also showed that rhIL-6 treatment increased EL expression between 4 and $12 \mathrm{~h}$ and peaked $8 \mathrm{~h}$ after treatment. This confirmed the time-dependent effects of IL-6 on EL expression in endothelial cells.

MAPK is an important intracellular signal transduction pathway. The MAPK signaling pathway can respond to various extracellular stimuli to mediate cell growth, differentiation and apoptosis, and is associated with endothelial dysfunction, inflammation, hypertension and vascular remodeling (25-28). 
The inhibition of p38 MAPK has been shown to be a clinical tool against inflammatory diseases, including chronic obstructive pulmonary disease (29). The p38 MAPK expression level increases in atherosclerosis, which may constitute a causative mechanism $(15,29)$. This may be one of the mechanisms for inflammation-induced atherosclerosis. Paravicini et al (29) found that the development of fibrosis can be inhibited by the inhibition of p38 MAPK by SB203580, which suggests that the signal is transmitted via the p38 MAPK pathway.

$\mathrm{NF}-\kappa \mathrm{B}$ is an important transcriptional regulatory factor, which responds to various cytokines. NF- $\mathrm{KB}$ is activated and enters the nucleus where it combines with specific DNA motifs and stimulates the expression of various genes. Chromatin immunoprecipitation and electrophoretic mobility shift assays have revealed that the EL gene has two NF- $\mathrm{KB}$ binding sites (30). The activation of NF- $\mathrm{KB}$ has also been observed in human and experimental atherosclerosis $(31,32)$. Cao et al (33) found that the progression of the instability of atherosclerotic plaques may be mediated by the NF- $\mathrm{KB}$ pathway.

In the current study, HUVECs were pretreated with a p38 MAPKs inhibitor (SB203580) or an NF-kB inhibitor (PDTC) prior to rhIL-6 treatment and the results showed that the pretreatment of HUVECs with either SB203580 or PDTC inhibited the IL-6-induced upregulation of EL expression. This therefore confirmed that IL-6 can regulate the expression of EL partly via the p38 MAPK and NF- $\mathrm{KB}$ signaling pathways.

In conclusion, the current study found that external addition of rhIL-6 upregulates the expression of EL in endothelial cells in a time-dependent manner and this upregulation can be partially inhibited by the p38 MAPK inhibitor (SB203580) or NF- $\mathrm{BB}$ inhibitor (PDTC). This in vitro study suggests that p38 MAPK and p65 NF- $\mathrm{KB}$ may participate in the process of IL-6-regulated expression of EL, and may be another mechanism by which IL-6 is involved in the pathogenesis of atherosclerosis. Although this study cannot be extrapolated for in vivo conditions, it provides evidence on a possible mechanism by which IL- 6 affects EL expression in endothelial cells. Thus, this study may provide a theoretical and experimental basis for future preventative treatments that target these factors specifically.

\section{Acknowledgements}

This study was supported by the Natural Science Foundation of Shandong Province (grant nos. Y2008C47, ZR2011HM086, ZR2014HM082 and ZR2011CM042).

\section{References}

1. Rattazzi M, Puato M, Faggin E, Bertipaglia B, Zambon A and Pauletto P: C-reactive protein and interleukin-6 in vascular disease: Culprits or passive bystanders? J Hypertens 21: 1787-1803, 2003.

2. Bernberg E, Ulleryd MA, Johansson ME and Bergström GM: Social disruption stress increases IL-6 levels and accelerates atherosclerosis in ApoE-/- mice. Atherosclerosis 221: 359-365, 2012.

3. Nishimoto N: Interleukin-6 in rheumatoid arthritis. Curr Opin Rheumatol 18: 277-281, 2006.

4. Barton BE: Interleukin-6 and new strategies for the treatment of cancer, hyperproliferative diseases and paraneoplastic syndromes. Expert Opin Ther Targets 9: 737-752, 2005.
5. Smolen JS and Maini RN: Interleukin-6: A new therapeutic target. Arthritis Res Ther 8: S5, 2006.

6. Genovese MC, McKay JD, Nasonov EL, Mysler EF, da Silva NA, Alecock E, Woodworth T and Gomez-Reino JJ: Interleukin- 6 receptor inhibition with tocilizumab reduces disease activity in rheumatoid arthritis with inadequate response to disease-modifying antirheumatic drugs: The tocilizumab in combination with traditional disease-modifying antirheumatic drug therapy study. Arthritis Rheum 58: 2968-2980, 2008.

7. Williams SC: First IL-6-blocking drug nears approval for rare blood disorder. Nat Med 19: 1193, 2013.

8. Jaye M, Lynch KJ, Krawiec J, Marchadier D, Maugeais C, Doan K, South V, Amin D, Perrone M and Rader DJ: A novel endothelial-derived lipase that modulates HDL metabolism. Nat Genet 21: 424-428, 1999.

9. Ishida T, Choi S, Kundu RK, Hirata K, Rubin EM, Cooper AD and Quertermous T: Endothelial lipase is a major determinant of HDL level. J Clin Invest 111: 347-355, 2003.

10. Gordon DJ and Rifkind BM: High-density lipoproteins-the clinical implications of recent studies. N Engl J Med 321: 1311-1316, 1989.

11. Broedl UC, Maugeais C, Millar JS, Jin W, Moore RE, Fuki IV, Marchadier D, Glick JM and Rader DJ: Endothelial lipase promotes the catabolism of ApoB-containing lipoproteins. Circ Res 94: 1554-1561, 2004.

12. Ishida T, Choi SY, Kundu RK, Spin J, Yamashita T, Hirata K, Kojima Y, Yokoyama M, Cooper AD and Quertermous T: Endothelial lipase modulates susceptibility to atherosclerosis in apolipoprotein-E-deficient mice. J Biol Chem 279: 45085-45092, 2004.

13. Jin W, Sun GS, Marchadier D, Octtaviani E, Glick JM and Rader DJ: Endothelial cells secrete triglyceride lipase and phospholipase activities in response to cytokines as a result of endothelial lipase. Circ Res 92: 644-650, 2003.

14. Choi SY, Hirata K, Ishida T, Quertermous T and Cooper AD: Endothelial lipase: A new lipase on the block. J Lipid Res 43: 1763-1769, 2002.

15. Han J, Lee JD, Bibbs L and Ulevitch RJ: A MAP kinase targeted by endotoxin and hyperosmolarity in mammalian cells. Science 265: 808-811, 1994.

16. Monaco C, Andreakos E, Kiriakidis S, Mauri C, Bicknell C, Foxwell B, Cheshire N, Paleolog E and Feldmann M: Canonical pathway of nuclear factor kappa B activation selectively regulates proinflammatory and prothrombotic responses in human atherosclerosis. Proc Natl Acad Sci 101: 5634-5639, 2004.

17. Annema W and Tietge UJ: Role of hepatic lipase and endothelial lipase in high-density lipoprotein-mediated reverse cholesterol transport. Nat Rev Drug Discov 4: 193-205, 2005.

18. Badellino KO, Wolfe ML, Rielly MP and Rader DJ: Endothelial lipase concentrations are increased in metabolic syndrome and associated with coronary atherosclerosis. PLoS Med 3: e22, 2006.

19. Cai G, He G and Qi C: The association between endothelial lipase 2384A/C gene polymorphism and acute coronary syndrome in a Chinese population. Mol Biol Rep 39: 9879-9884, 2012.

20. Singaraja RR, Sivapalaratnam S, Hovingh K, Dubé MP, Castro-Perez J, Collins HL, Adelman SJ, Riwanto M, Manz J, Hubbard B, et al: The impact of partial and complete loss of function mutations in endothelial lipase on high-density lipoprotein levels and functionality in humans. Circ Cardiovasc Genet 6: 54-62, 2013.

21. Fang YQ, Huang L and Li AM: Correlation between the expression of endothelial lipase in CECs and the improved jeopardy score in CAD patients. Chinese Journal of Critical Care Medicine 28: 5-8, 2008.

22. Hirata K, Ishida T, Matsushita H, Tsao PS and Quertermous T: Regulated expression of endothelial cell-derived lipase. Biochem Biophys Res Commun 272: 90-93, 2000.

23. Jin W, Sun GS, Marchadier D, Octtaviani E, Glick JM and Rader DJ: Endothelial cells secrete triglyceride lipase and phospholipase activities in response to cytokines as a result of endothelial lipase. Circ Res 92: 644-650, 2003.

24. Badellino KO, Wolfe ML, Reilly MP and Rader DJ: Endothelial lipase is increased in vivo by inflammation in humans. Circulation 117: 678-685, 2008.

25. Paradis ME, Badellino KO, Rader DJ, Deshaies Y, Couture P, Archer WR, Bergeron N and Lamarche B: Endothelial lipase is associated with inflammation in humans. J Lipid Res 47: 2808-2813, 2006. 
26. Yogi A, Callera GE, Aranha AB, Antunes TT, Graham D, McBride M, Dominiczak A and Touyz RM Sphingosine-1-phosphate-induced inflammation involves receptor tyrosine kinase transactivation in vascular cells: Upregulation in hypertension. Hypertension 57: 809-818, 2011

27. Gerthoffer WT: Mechanisms of vascular smooth muscle cell migration. Circ Res 100: 607-621, 2007.

28. Jacobsen JC, Mulvany MJ and Holstein-Rathlou NH: A mechanism for arteriolar remodeling based on maintenance of smooth muscle cell activation. Am J Physiol Regul Integr Comp Physiol 294: R1379-R1389, 2008.

29. Paravicini TM, Montezano AC, Yusuf H and Touyz RM: Activation of vascular p38 MAPK by mechanical stretch is independent of c-Src and NADPH oxidase: Influence of hypertension and angiotensin II. J Am Soc Hypertension 6: 169-178, 2012.
30. Oeckinghaus A and Ghosh S: The NF-kappaB family of transcription factors and its regulation. Cold Spring Harb Perspect Biol 1: a000034, 2009.

31. Brand K, Page S, Rogler G, Bartsch A, Brandl R, Knuechel R, Page M, Kaltschmidt C and Baeuerle PA: Activated transcription factor nuclear factor-kappa $\mathrm{B}$ is present in the atherosclerotic lesion. J Clin Invest 97: 1715-1722, 1996.

32. Hajra L, Evans AI, Chen M, Hyduk SJ, Collins T and Cybulsky MI: The NF-kappa B signal transduction pathway in aortic endothelial cells is primed for activation in regions predisposed to atherosclerotic lesion formation. Proc Natl Acad Sci USA 97: 9052-9057, 2000.

33. Cao Y, Zhou X, Liu H, Zhang Y, Yu X and Liu C: The NF- $\kappa B$ pathway: Regulation of the instability of atherosclerotic plaques activated by Fg, Fb and FDPs. Mol Cell Biochem 383: 29-37, 2013. 